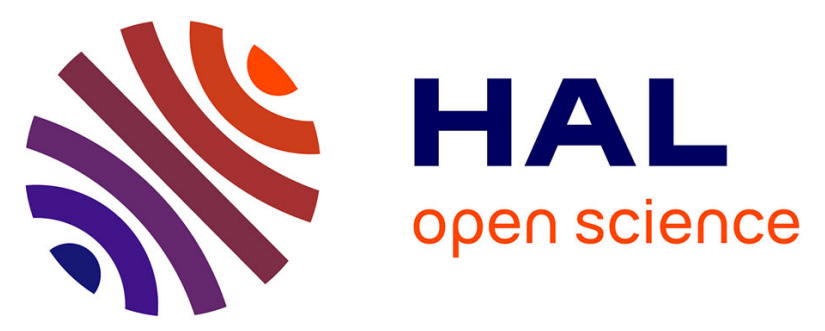

\title{
Environmental Nanoparticle-Induced Toughening and Pinning of a Growing Crack in a Biopolymer Hydrogel
}

O. Ronsin, I. Naassaoui, A. Marcellan, T. Baumberger

\section{To cite this version:}

O. Ronsin, I. Naassaoui, A. Marcellan, T. Baumberger. Environmental Nanoparticle-Induced Toughening and Pinning of a Growing Crack in a Biopolymer Hydrogel. Physical Review Letters, 2019, 123

(15), pp.158002. 10.1103/PhysRevLett.123.158002 . hal-02498100

\section{HAL Id: hal-02498100 \\ https://hal.science/hal-02498100}

Submitted on 4 Mar 2020

HAL is a multi-disciplinary open access archive for the deposit and dissemination of scientific research documents, whether they are published or not. The documents may come from teaching and research institutions in France or abroad, or from public or private research centers.
L'archive ouverte pluridisciplinaire HAL, est destinée au dépôt et à la diffusion de documents scientifiques de niveau recherche, publiés ou non, émanant des établissements d'enseignement et de recherche français ou étrangers, des laboratoires publics ou privés. 


\title{
Environmental Nanoparticle-Induced Toughening and Pinning of a Growing Crack in a Biopolymer Hydrogel
}

\author{
O. Ronsin, ${ }^{1}$ I. Naassaoui, ${ }^{1,2}$ A. Marcellan, ${ }^{3}$ and T. Baumberger ${ }^{1}$ \\ ${ }^{1}$ Institut des NanoSciences de Paris, Sorbonne University, CNRS, F-75005 Paris, France \\ ${ }^{2}$ Laboratoire de Physique de la Matière Molle et de la Modélisation Électromagnétique, \\ Université de Tunis, El Manar, 2092 Tunis, Tunisia \\ ${ }^{3}$ Laboratoire Sciences et Ingénierie de la Matière Molle, ESPCI Paris, PSL University, \\ Sorbonne University, CNRS, F-75005 Paris, France
}

(Received 19 April 2019; published 8 October 2019)

\begin{abstract}
We study the interplay between a crack tip slowly propagating through a hydrogel and nanoparticles suspended in its liquid environment. Using a proteinic gel enables us to tune the electrostatic interaction between the network and silica colloids. Thereby, we unveil two distinct, local toughening mechanisms. The primary one is charge independent and involves the convective building of a thin particulate clog, hindering polymer hydration in the crack process zone. When particles and network bear opposite charges, transient adhesive bonding superimposes, permitting the remarkable pinning of a crack by a liquid drop.
\end{abstract}

DOI: 10.1103/PhysRevLett.123.158002

Introduction.-Over the past decades, polymer hydrogels have reached the status of genuine "structural" materials for their applications in tissue engineering [1], wound dressing, and drug delivery [2], where, in addition to stringent mechanical strength requirements in terms of fracture toughness and flaw tolerance, they are expected to accommodate the osmotically active, physiological environment. These soft solids deserve a dedicated chapter in fracture mechanics owing to their high stretchability [3-5] and biphasic structure [6-10] which challenge both the $L$ and $E$ of the standard Linear Elastic Fracture Mechanics framework [11]. Indeed, the tip of a crack growing through a hydrogel is an elastically blunted [3], highly stretched zone [4], widely exposed to the outer space and extremely prone to exchange solvent and solute with its liquid environment. Small molecules readily diffuse into the crack tip and interfere with the rupture process [12-14]. This usually results in a weakening of the crack, i.e., a lowering of the fracture energy $\Gamma$, a soft matter counterpart to stress corrosion in hard, brittle solids $[15,16]$. Although desirable, the opposite effects, i.e., crack toughening and pinning, remain an elusive goal [7].

Because of their high surface activity and diameters commensurate with polymer network mesh sizes, nanoparticles (NPs) can interact synergistically with soft hydrogels $[17,18]$. Recently, the ability of silica NP solutions to promote adhesion between two wet hydrogel or biological tissue surfaces has been demonstrated [19-21] and applied in surgery [22]. Strong healing is obtained, provided that (i) the network bears an electric charge opposite to that of NPs, and (ii) the excess solvent is squeezed out of the interfacial joint, so that particles come into intimate contact with the network. The latter requirement limits the healing time (on the order of tens of seconds [23]) for emergency suture of macroscopic wounds. Now, consider a crack in a hydrogel, propagating at velocity $V$. Polymer chains dissipating energy are located in a process zone (PZ) extending over $d$ ahead of the tip [12]. The dwell time of a chain in the $\mathrm{PZ}$ is therefore $\tau \simeq d / V$. With $d \simeq 100 \mathrm{~nm}$ and $V \simeq 1 \mathrm{~cm} \mathrm{~s}^{-1}$, relevant for this study, $\tau$ is as short as $10^{-5} \mathrm{~s}$, during which NPs cannot diffuse over more than a radius in free water, even less into the crowded PZ. This seems to hinder a priori any efficient NP-network interplay. Fortunately, hydrogels are poroelastic soft solids, i.e., nanosponges able to absorb or expel solvent in response to a chemical or mechanical stimulus $[10,24,25]$. The corresponding "breathing" flow was shown to advect efficiently particles onto the surface [26] or even into the bulk [27] of the network, depending on their diameter relative to the mesh size. Besides, even in a swollen gel at osmotic equilibrium, the high stress gradients prevailing in the tip vicinity induce a solvent flow in a region of typical size $\mathcal{L}_{\text {poro }}=D_{\text {coll }} / V$ with $D_{\text {coll }}$ the network or solvent diffusion coefficient $[7,28]$. Motivated by the idea of poroelastically driven, localized NP flow, we have systematically assessed the effect of wetting a slow crack with a NP solution. Remarkably, we have found two original and efficient toughening mechanisms, opening a new perspective for the fracture physics of ultrastretchable solids.

Experiments.-Experiments are performed with gelatin gels and silica nanoparticles. The latter are commercial Ludox solutions of two grades, TM and AM, diluted with $50 \mathrm{mM} \mathrm{NaCl}$ to reach a volume fraction $\phi$. The nominal average particle diameters $2 R$ are, respectively, 22 and $12 \mathrm{~nm}$ for TM and AM. These silica NPs remain negatively charged under usual conditions $(4<p \mathrm{H}<10.5)$. Depending 


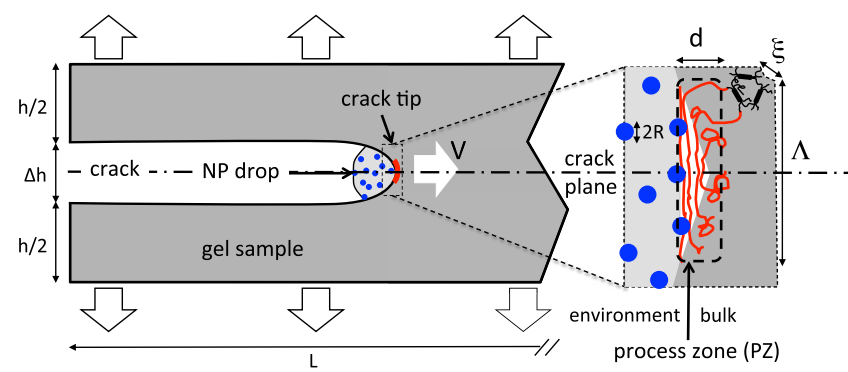

FIG. 1. Left: Schematic representation of a crack propagating a velocity $V$ in the midplane of a stretched gel slab. A drop of NP solution moves along with the crack tip. The amount of elastic energy $\mathcal{G}$ released by opening a unit area of crack surface is imposed by the fixed grip displacement (vertical arrows). The crack opening $\Delta h$ is typically a few millimeters. Right: Enlargement of the tip, showing the PZ of depth $d$ and height $\Lambda$, made of polymer chains (red online) pulled out taut from the gel matrix. Triple-helix crosslinks, distant of $\xi$ (mesh size) on average, are unzipped at the frontier between the fibrillar zone and the bulk. Because of the nonlinear elastic response of the surrounding matrix, the $\mathrm{PZ}$ is quasi-2D (aspect ratio $d / \Lambda \ll 1$ ), perpendicular to the crack plane. Nanoparticles (blue online) have diameters $2 R \gtrsim \xi$.

on the denaturation process of the native collagen, gelatin comes in two types, customly referred to as $A$ and $B$, differing in their isoelectric points pI, respectively, 5 and 9 . Both yield thermoreversible gels with a $p \mathrm{H} \simeq 6$ in their preparation state so that type- $A$ networks are positively charged while type- $B$ ones bear a weak negative charge. We used gels of both types with a $5 \mathrm{wt} \%$ gelatin concentration, yielding a shear modulus $G \simeq 2.5 \mathrm{kPa}$ and, hence, a network mesh size [12] $\xi \simeq 12 \mathrm{~nm} \lesssim 2 R$.

Long gel slabs (length $L=300 \mathrm{~mm}$, height $h=30 \mathrm{~mm}$, and thickness $e=10 \mathrm{~mm}$ ), prepared as previously described [12], are stretched uniformly by $\Delta h<8 \mathrm{~mm}$ along their height (Fig. 1). A slow-growing crack feels a constant energy release rate $\mathcal{G}(\Delta h)$, estimated as usual in the "pure-shear" geometry [12]. The steady crack velocity $V$ adjusts itself to fulfill Griffith's criterion $[4,29]: \Gamma(V)=$ $\mathcal{G}(\Delta h)$ with $\Gamma$ the rate-dependent fracture energy. $V$ is measured by image processing a movie of the crack tip with spatial resolution $30 \mu \mathrm{m}$ at a rate of 20 frames per second. A drop of NP solution is introduced in the crack tip opening, thereby providing a particle reservoir. Capillary pressure induced by the wetting drop contributes negligibly to $\mathcal{G}$ (see Supplemental Material [30]). Three tip environments are referred to in the following: $d r y$ when the crack opens in air, wet when it is wetted by a drop of pure solvent, and $\mathrm{NP} \phi$ when it is wet by a solution of NPs at a volume fraction $\phi$. The outcome of an experimental run in the NP $\phi$ state is the fracture energy $\Gamma^{\mathrm{NP}}(V, \phi)$ over two decades of velocities (typically, $0.1-10 \mathrm{~mm} / \mathrm{s}$ ) swept by changing $\Delta h$.

Qualitative behavior.-Figure 2 illustrates the fate of a dry, steady crack, after modification of its liquid environment. As previously reported [12], a crack wet by pure solvent speeds up. However, when wetted by a diluted NP

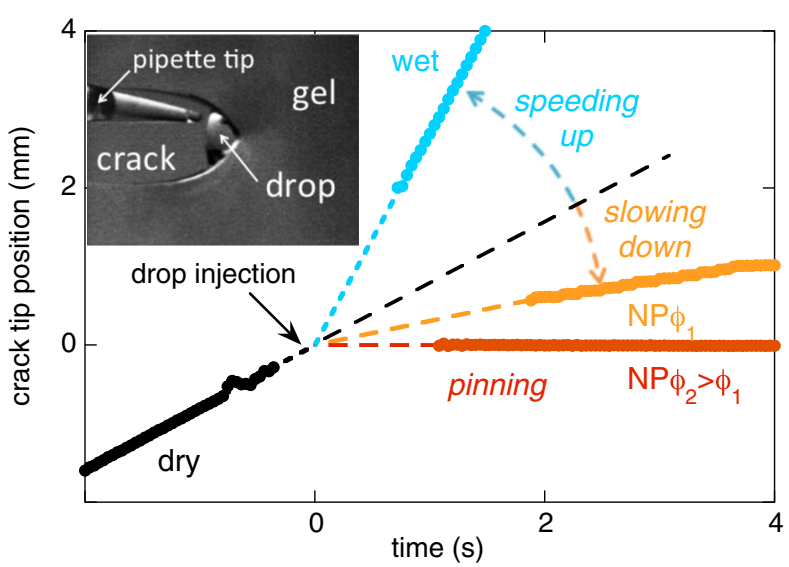

FIG. 2. Time-dependent position of an initially dry, steady crack in a gelatin $A$ hydrogel. Upon wetting (at $t=0$ ) its tip by a drop of AM Ludox solution (see the inset) at a fixed grip, a new steady state is reached. Depending on the silica volume fraction $\phi$, the crack speeds up $(\phi=0)$, slows down $\left(\phi_{1}=5 \%\right)$, or even gets pinned $\left(\phi_{2}=16 \%\right)$. (See Supplemental Movies [30].)

solution, it slows down markedly and even stops on the spot, i.e., gets pinned, at a high enough NP content (see also Supplemental Movies [30]). Nanoparticles have therefore a strong toughening effect on the crack which is able to counterbalance the solvent-induced weakening. Accordingly, in the following, we will investigate the effect of nanoparticle solutions with reference to the wet case. We will seize on the opportunity of building networks with similar structure but opposite electric charges to investigate separately what we will refer to as the "repulsive" and "adhesive" cases.

Repulsive case.-We first address the case of negatively charged gelatin $B$ networks. Quite unexpectedly, even in this a priori unfavorable case, we observe a strong effect of the like-charged NPs. As seen in Fig. 3(a), whatever $\phi$, the fracture energy curves of $\mathrm{NP} \phi$ cracks $\Gamma^{\mathrm{NP}}(V, \phi)$ lie above the wet crack one $\Gamma^{\text {wet }}(V)$ over the whole velocity range. We refer to this phenomenon as the primary toughening effect of NPs and characterize it by the energy shift $\Gamma^{\mathrm{NP}}-\Gamma^{\text {wet }}[$ Fig. 3(b)]. The more concentrated the solution, the stronger the toughening.

It is enlightening to use the fracture energy of the dry crack as a benchmark against which to assess the toughening effects of NPs (Fig. 3). Upon increasing $\phi$, not only are the $\Gamma^{\mathrm{NP}}(V, \phi)$ curves shifted towards higher energies, but they also progressively depart from the linearity of $\Gamma^{\text {wet }}(V)$ and markedly bend at low velocities (typically in the sub- $\mathrm{mm} \mathrm{s}^{-1}$ range), thus acquiring a characteristic feature of $\Gamma^{\text {dry }}(V)$. In other words, cracks wetted by a NP solution mimic the dry crack state. This paradoxical statement calls for details on the mechanisms underlying the dry and wet crack propagation.

In the case of gelatin gels, a decade of experimental work has led to the following simple picture $[12,28,31]: \Gamma^{\text {wet }}(V)$ is 

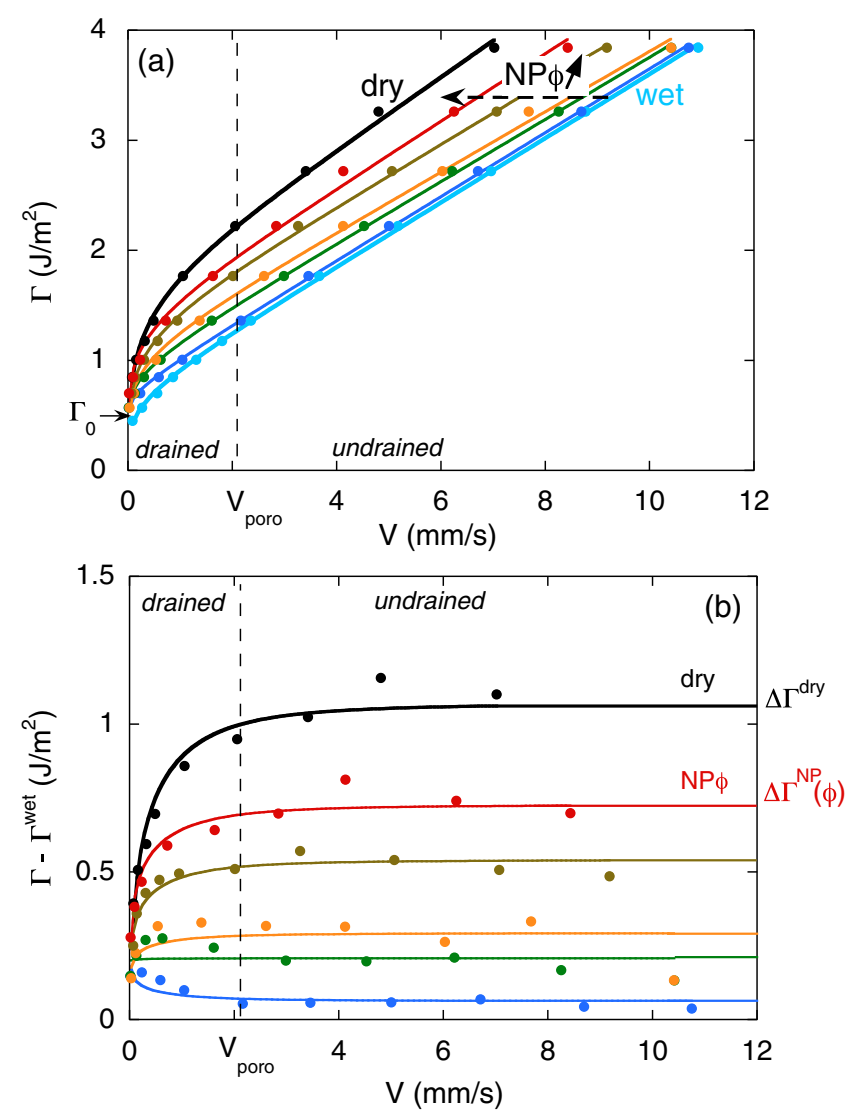

FIG. 3. (a) Rate-dependent fracture energies $\Gamma(V)$ of cracks in a weakly negatively charged gelatin $B$ gel with different tip environments: wet (lower curve), dry (upper curve), and NP $\phi$ (intermediate curves) with negatively charged TM-grade silica nanoparticles of volume fraction increasing from right to left: $\phi=4.8 \%, 10 \%, 16 \%, 23 \%, 31 \%$. Curves are guides for the eyes. (b) Fracture energy shifts with respect to the wet case. The poroelastic velocity $V_{\text {poro }} \simeq 2 \mathrm{~mm} \mathrm{~s}^{-1}$ and the associated drained and undrained crack propagation regimes are defined in the text. In the undrained limit, the energy shift reaches a plateau $\Delta \Gamma^{\mathrm{dry}}$ for a dry crack and $\Delta \Gamma^{\mathrm{NP}}(\phi)$ for a $\mathrm{NP} \phi$ crack.

quasilinear. Its extrapolated stationary value $\Gamma_{0}$ corresponds to the energy threshold to unzip triple-helix, physical (noncovalent) cross-links. Its rate dependence stems from the viscous dissipation associated with pulling a chain out of the gel matrix. At large enough velocities, $\Gamma^{\text {dry }}(V)$ is linear with the same slope as $\Gamma^{\text {wet }}(V)$. It is merely translated by $\Delta \Gamma^{\mathrm{dry}}>0$, which amounts to the energy cost for exposing chains to air. For $V<V_{\text {poro }}=D_{\text {coll }} / \xi \simeq 2 \mathrm{~mm} \mathrm{~s}^{-1}$, the poroelastic diffusive length being larger than the mesh size $\xi$, the gel can be partially drained, i.e., solvent flow through the network in the tip vicinity so as to rehydrate the PZ. This mitigates the effect of the dry tip environment, hence the observed gradual inflexion of the dry fracture energy towards the wet one.

All these characteristic features of dry cracks appear to be shared by NP-wetted cracks. Accordingly, we propose that the primary effect of NPs is to hinder the access of the drop solvent to the pulled-out chains, i.e., to $c \log$ the PZ. In the undrained limit $\left(V>V_{\text {poro }}\right)$, any bulk poroelastic flow is precluded, and the only source of hydration is environmental. In the following, we will restrict our analysis to this simpler case. Remarkably, the effect of NPs in this regime amounts to a mere $V$-independent energy shift:

$$
\Gamma^{\mathrm{NP}}(V, \phi)-\Gamma^{\text {wet }}(V) \underset{V>V_{\text {poro }}}{\longrightarrow} \Delta \Gamma^{\mathrm{NP}}(\phi) .
$$

This is all the more unexpected, since at large velocities the lifetime of the PZ, $\tau=d / V$, during which NPs can diffuse and interact with active chains, vanishes. This is a strong hint that an advective solvent flow pervades the $\mathrm{PZ}$ at a rate increasing with $V$, thereby counteracting the unfavorable lifetime effect.

In order to put this on an assessable footing, we resort to a schematic picture of the PZ in a physical gel (Fig. 1), made legitimate by a series of experimental studies [12-14,28,31]. Note that, although its fibrillar structure (taut chains drawn plastically from the bulk) is reminiscent of a craze in a polymer glass [32], the PZ extends perpendicular to the crack plane rather than along it, due to the high stretchability of hydrogels [4]. It is therefore described as a thin skin of height $\Lambda$ and depth $d \ll \Lambda$ ( $d$ was estimated of the order of $\lambda / 10 \simeq 100 \mathrm{~nm}$ from crack wetting experiments [12]). Owing to this quasi-2D geometry, we lump all the details of the NP-PZ interaction into a single parameter, namely, the areal fraction $\alpha$ of the $\mathrm{PZ}$ that is clogged by particles.

The steady state value of $\alpha$ results from a balance between the volume flux of particles advected by the solvent and the surface flux due to the crack opening which carries adsorbed particles away from the tip. A scaling estimate of $\alpha$ boils down from the following arguments. Pulling taut un-crosslinked chains out of the gel matrix opens free volume at a rate of $\sim \Lambda V$ per unit crack width. The negative chemical potential of the solvent in the presence of soluble polymers drives it towards the PZ. For $V \gg V_{\text {poro }}$, the flow is localized in the drop $\sim \Lambda$ around the tip. It therefore advects NPs at a rate of $\sim \phi V / R^{3}$. Because of the crack advance, the $\mathrm{PZ}$ is steadily refreshed over a time $\sim \tau=d / V$, thereby removing the clog particles at a rate $\sim \tau^{-1} \alpha / R^{2}$. Upon balancing both rates, $V$ cancels out:

$$
\alpha=C \phi d / R
$$

with $C$ a geometrical constant of order unity (see Supplemental Material [30] for a slightly more elaborate derivation).

Finally, we connect particle dynamics and fracture mechanics by assuming that a fraction $\alpha<1$ of the $\mathrm{PZ}$ is clogged and that the corresponding fracture energy shift is

$$
\Delta \Gamma_{\text {clog }}(\phi)=\alpha(\phi) \Delta \Gamma^{\text {dry }} .
$$

The predicted $\Delta \Gamma_{\text {clog }}$ must now be compared to the experimental $\Delta \Gamma^{\mathrm{NP}}$. Beyond the already mentioned $V$ 


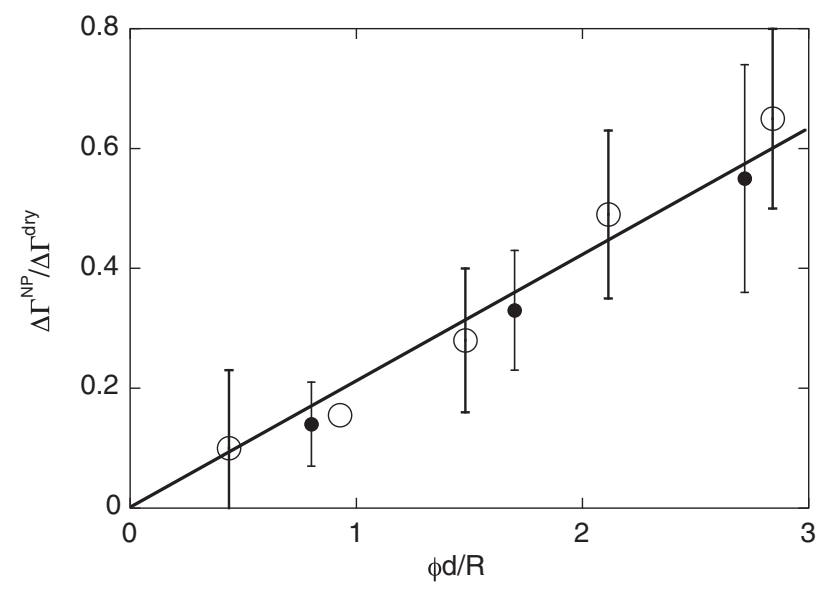

FIG. 4. Reduced toughening parameter in the undrained regime for gelatin $B$ gels (repulsive case) plotted with respect to the dimensionless parameter $\phi d / R$ with $d=100 \mathrm{~nm}$ the depth of the process zone (as estimated in Ref. [12]) and nanoparticle radii: $R=6 \mathrm{~nm}$ for AM particles (small bullets) and $R=11 \mathrm{~nm}$ for TM particles (big circles). Bars indicate the standard deviation over 3-4 different samples. The linear fit corresponds to the clog model $\Delta \Gamma_{\text {clog }}=C \phi d / R$ with $C \simeq 0.2$.

independence, the data in Fig. 4 are in full agreement with a linear dependence of $\Delta \Gamma^{\mathrm{NP}} / \Delta \Gamma^{\mathrm{dry}}$ on $\phi d / R$, for the two types of NPs (with radii in a ratio 2:1). The scaling constant $C \simeq 0.2$ being of the order of unity lends strong support to our scenario. Once sucked into the PZ, particles are kept in place by the negative pore pressure so that their pore blocking efficiency should depend more on their size $(2 R \gtrsim \xi)$ than on their electric charge.

Adhesive case.-We are now in a position to investigate the case where the network is positively charged (gelatin $A$ ) and seek for an adhesive contribution proper. Figure 5 illustrates the toughening effect of TM particles. Although it looks qualitatively similar to those of Fig. 3(b), characterized by a rate-independent asymptotic value $\Delta \Gamma^{\mathrm{NP}}(\phi)$ in the undrained limit, a closer inspection reveals that, at a large enough particle fraction, $\Delta \Gamma^{\mathrm{NP}}>\Delta \Gamma^{\text {dry }}$ over the experimental $V$ range. Since a crack can hardly be "dryer than dry," this clearly makes it legitimate for us to define a residual fracture energy:

$$
\Delta \Gamma_{\text {adh }}(\phi)=\Delta \Gamma^{\mathrm{NP}}-\Delta \Gamma_{\text {clog }} .
$$

We computed $\Delta \Gamma_{\text {adh }}$ using the value of the geometric constant $C=0.2$ fitting the data in Fig. 4, assuming that the structure of both gels of comparable elastic moduli were similar. As seen in Fig. 5(b), the adhesive toughening is a remarkably efficient effect: $\Delta \Gamma_{\text {adh }}(\phi)$ increases slightly and saturates to $\simeq 3 \Gamma_{0}$ with $\Gamma_{0}$ the wet crack threshold [see Fig. 5(c)]. This suggests the formation of a NP-chain transient network $[23,33,34]$ in the PZ, where particles act as physical cross-links of strength comparable to that of the native triple-helix ones. Moreover, the rate
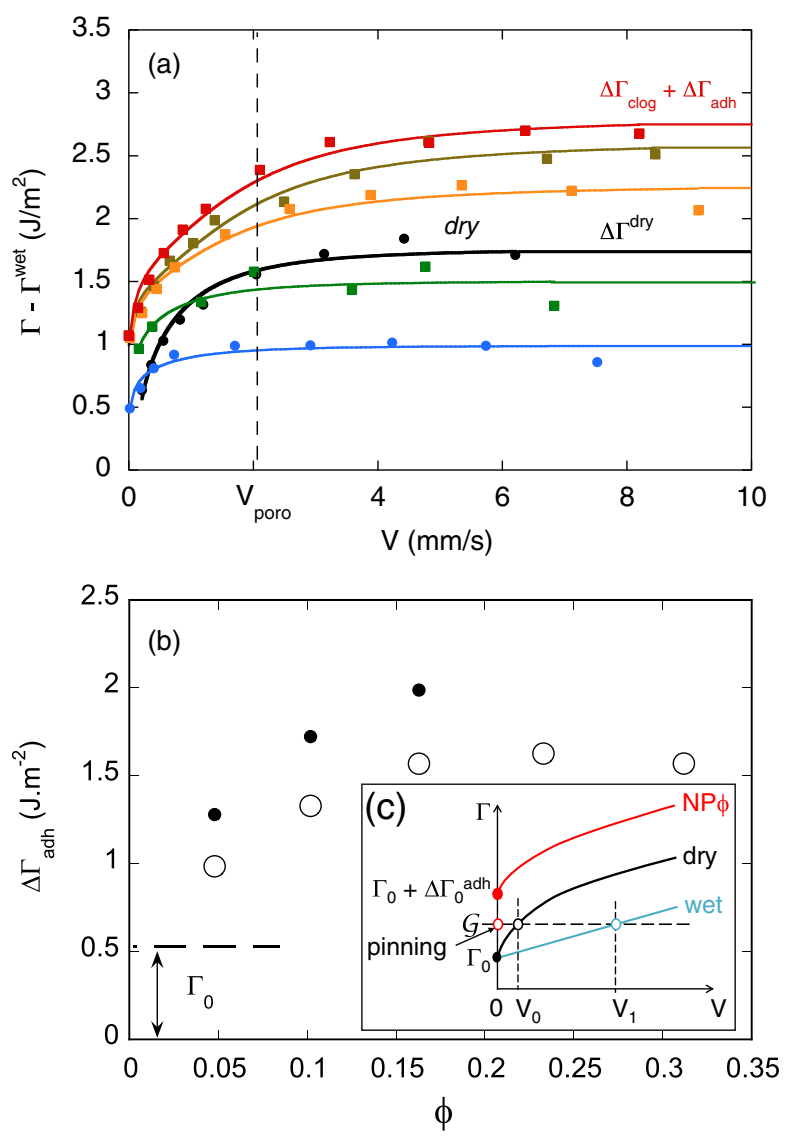

FIG. 5. (a) Relative fracture energy shift for gelatin $A$ gels and TM nanoparticles (adhesive case). The data for NP $\phi$ cracks are ordered from bottom to top with increasing silica volume fraction. Curves are guides for the eyes. The values of $\phi$ and colors (online) are the same as in Fig. 3. Note that the dry crack curve stands in an intermediate position. (b) Adhesive contribution to crack toughening $\Delta \Gamma_{\text {adh }}$ (see the text) with respect to the silica volume fraction $\phi$, for AM (small bullets) and TM (big circles) particles. (c) Schematic representation of the fracture energy of a crack in its three tip states (dry, wet, and NP $\phi$ in the adhesive regime) showing the geometric construct of the crack velocity at constant energy release rate $\mathcal{G}$. A dry crack running at velocity $V_{0}$ speeds up to $V_{1}$ upon wetting by pure solvent but gets pinned upon wetting by a suitably concentrated NP solution (see Fig. 2 and Supplemental Movies [30]).

independence of the effect means that such noncovalent but strong links form within less than $d / V$, typically a few $10^{-5} \mathrm{~s}$, while triple helices require hours of aging to reach their full strength $\Gamma_{0}$ (see section methods in Supplemental Material [30]). This is a hint that, beyond the favorable charge state, the commensurability of $R$ and $\xi$ concur to achieve optimal interactions.

Finally, we comment on the condition for crack pinning (Fig. 2). At constant energy release rate $\mathcal{G}$, a dry crack will slow down upon wetting if the $\mathrm{NP} \phi$ fracture energy curve lies above the dry one [Fig. 5(c)]. This is achievable only in the adhesive case. If the corresponding threshold energy 
$\Gamma_{0}+\Delta \Gamma_{0}^{\text {adh }}$ is such that $\Gamma_{0}<\mathcal{G}<\Gamma_{0}+\Delta \Gamma_{0}^{\text {adh }}$, the wet crack becomes subcritical and stops propagating: It gets pinned.

Conclusion.-We have demonstrated the fast and focused action of nanoparticles on cracks in an important class of soft materials, hydrogels being commonly considered as proxies for soft biological tissues. Passive targeting [35], i.e., the ability of nanoparticles to interact specifically with organs [36], or lesions [37] without biochemical labeling, on the sole basis of their size and charge is an important, though poorly understood, aspect of nanomedicine. Our study reveals how a strongly inhomogeneous strain field couples with the porous nature of the gel to trigger a self-targeting, efficient healing action of nanoparticles on short timescales precluding bulk poroelastic flow to occur. This confirms the fundamental interest of studying stress-induced, multiple-scale water flux in tissues and mimicking constructs $[10,38,39]$.

We thank S. Cohen-Addad for the careful reading of the manuscript. I. N. received a scholarship from the government of Tunisia.

[1] S. Naahidi, M. Jafari, M. Logan, Y. Wang, Y. Yuan, H. Bae, B. Dixon, and P. Chen, Biotechnology advances 35, 530 (2017).

[2] J. S. Boateng, K. H. Matthews, H. N. Stevens, and G. M. Eccleston, J. Pharm. Sci. 97, 2892 (2008).

[3] C.-Y. Hui, A. Jagota, S. Bennison, and J. Londono, Proc. R. Soc. A 459, 1489 (2003).

[4] V. R. Krishnan, C. Y. Hui, and R. Long, Langmuir 24, 14245 (2008).

[5] J. Tang, J. Li, J. J. Vlassak, and Z. Suo, Extreme Mech. Lett. 10, 24 (2017).

[6] C.-Y. Hui, R. Long, and J. Ning, J. Appl. Mech. 80, 021014 (2013).

[7] G. Noselli, A. Lucantonio, R. M. McMeeking, and A. DeSimone, J. Mech. Phys. Solids 94, 33 (2016).

[8] N. Bouklas and R. Huang, Soft Matter 8, 8194 (2012).

[9] Y. Yu, C. M. Landis, and R. Huang, J. Mech. Phys. Solids 118, 15 (2018).

[10] A. E. Ehret, K. Bircher, A. Stracuzzi, V. Marina, M. Zündel, and E. Mazza, Nat. Commun. 8, 1002 (2017).

[11] K. B. Broberg, Cracks and Fracture (Elsevier, New York, 1999).

[12] T. Baumberger, C. Caroli, and D. Martina, Nat. Mater. 5, 552 (2006).

[13] T. Baumberger and O. Ronsin, Eur. Phys. J. E 31, 51 (2010).

[14] T. Baumberger and O. Ronsin, Biomacromolecules 11, 1571 (2010).

[15] O. L. Anderson and P. C. Grew, Rev. Geophys. 15, 77 (1977).
[16] J. Williams and G. Marshall, Proc. R. Soc. A 342, 55 (1975).

[17] P. Thoniyot, M. J. Tan, A. A. Karim, D. J. Young, and X. J. Loh, Adv. Sci. 2, 1400010 (2015).

[18] S. Merino, C. Martin, K. Kostarelos, M. Prato, and E. Vazquez, ACS Nano 9, 4686 (2015).

[19] S. Rose, A. Prevoteau, P. Elzière, D. Hourdet, A. Marcellan, and L. Leibler, Nature (London) 505, 382 (2014).

[20] H. Abe, Y. Hara, S. Maeda, and S. Hashimoto, J. Phys. Chem. B 118, 2518 (2014).

[21] J.-H. Kim, H. Kim, Y. Choi, D. S. Lee, J. Kim, and G.-R. Yi, ACS Appl. Mater. Interfaces 9, 31469 (2017).

[22] A. Meddahi-Pellé, A. Legrand, A. Marcellan, L. Louedec, D. Letourneur, and L. Leibler, Angew. Chem., Int. Ed. 53, 6369 (2014).

[23] S. Rose, A. Dizeux, T. Narita, D. Hourdet, and A. Marcellan, Macromolecules 46, 4095 (2013).

[24] M. Doi, J. Phys. Soc. Jpn. 78, 052001 (2009).

[25] W. Hong, X. Zhao, J. Zhou, and Z. Suo, J. Mech. Phys. Solids 56, 1779 (2008).

[26] F. Boulogne, F. Ingremeau, J. Dervaux, L. Limat, and H. A. Stone, Europhys. Lett. 112, 48004 (2015).

[27] V. Pardo-Yissar, R. Gabai, A. Shipway, T. Bourenko, and I. Willner, Adv. Mater. 13, 1320 (2001).

[28] I. Naassaoui, O. Ronsin, and T. Baumberger, Extreme Mech. Lett. 22, 8 (2018).

[29] D. Maugis, Contact, Adhesion and Rupture of Elastic Solids (Springer Science \& Business, New York, 2013), Vol. 130.

[30] See Supplemental Material at http://link.aps.org/supplemental/ 10.1103/PhysRevLett.123.158002 for methods, supplementary discussion and movies.

[31] T. Baumberger, C. Caroli, and D. Martina, Eur. Phys. J. E 21, 81 (2006).

[32] C. Hui, A. Ruina, C. Creton, and E. Kramer, Macromolecules 25, 3948 (1992).

[33] K. Haraguchi, K. Uyama, and H. Tanimoto, Macromol. Rapid Commun. 32, 1253 (2011).

[34] A. K. Gaharwar, C. P. Rivera, C.-J. Wu, and G. Schmidt, Acta Biomater. 7, 4139 (2011).

[35] S. Gioux, H. S. Choi, and J. V. Frangioni, Mol. Imaging 9, 7290 (2010).

[36] L. Balogh, S. S. Nigavekar, B. M. Nair, W. Lesniak, C. Zhang, L. Y. Sung, M. S. Kariapper, A. El-Jawahri, M. Llanes, B. Bolton et al., Nanomedicine 3, 281 (2007).

[37] G. V. Kolmakov, R. Revanur, R. Tangirala, T. Emrick, T. P. Russell, A. J. Crosby, and A. C. Balazs, ACS Nano 4, 1115 (2010).

[38] A. Malandrino and E. Moeendarbary, in Encyclopedia of Biomedical Engineering, edited by R. Narayan (Elsevier, Oxford, 2019), pp. 238-245.

[39] P. A. Netti, L. T. Baxter, Y. Boucher, R. Skalak, and R. K. Jain, AIChE J. 43, 818 (1997). 